\title{
The effect of orography on the global atmospheric angular momentum and the general circulation
}

\author{
Oliver J. STENZEL ${ }^{1}$ and Jin-SONG VON STORCH ${ }^{* 2}$ \\ ${ }^{1}$ Max-Planck-Institut für Sonnensystemforschung, Katlenburg-Lindau, Germany \\ ${ }^{2}$ Max-Planck-Institut für Meteorologie, Hamburg, Germany
}

(Manuscript received October 13, 2004; in revised form May 10, 2005; accepted May 12, 2005)

\begin{abstract}
Orographic features affect the atmospheric circulation not only locally but also globally. In this paper, the latter is quantified in terms of the global relative angular momentum in a series of numerical experiments using the Portable University Model of the Atmosphere (PUMA). Two effects of an orographic barrier on the atmospheric relative angular momentum are identified. On the one hand, an orographic barrier acts to break the zonal symmetry of the circulation. Because of the symmetry breaking, the energy inputed is stored not only in the time-mean part, but also in the time-varying part of the zonal flow, leading to a reduction in the mean and an enhancement in the variance of the relative angular momentum. On the other hand, orographic features lead also to changes in the eddy momentum fluxes, which are necessary to balance the mountain torque. These eddy fluxes induce an increase of global relative angular momentum through a strengthening and an equatorward shift of the zonal flow. The second effect prevents a further decrease in the global relative angular momentum with an increase in the height of the barrier.
\end{abstract}

\section{Zusammenfassung}

Die Orographie der Erdoberfläche hat sowohl regionale als auch globale Auswirkung auf die atmosphärische Zirkulation. In dieser Arbeit wird die letztere, unter Betrachtung des globalen relativen Drehimpulses, anhand von numerischen Experimenten mit dem PUMA Modell untersucht. Zwei Effekte werden identifiziert. Zum einen verursacht ein Hindernis eine Unterbrechung der zonalen Symmetrie. Dadurch wird die der PUMAAtmosphäre zugefügte Energie nicht nur in dem mittleren, sondern auch in dem zeitlich variierenden Zonalstrom gespeichert, was zu einer Reduzierung des Mittelwerts und gleichzeitig zu einer Zunahme in der Varianz des globalen relativen Drehimpulses führt. Zum anderen ruft ein Hindernis Änderung im Eddyimpulsfluss hervor, die notwendig ist, um das Gebirgsmoment auszugleichen. Eine solche Änderung führt zu einer Zunahme des mittleren relativen Drehimpulses der Atmosphäre durch eine Verstärkung und eine Verlagerung des Zonalstroms äquatorwärts. Der zweite Effekt verhindert ein weitere Abnahme des relativen Drehimpulses bei einer Erhöhung des Hindernisses.

\section{Introduction}

The general circulation of the atmosphere, characterized by strong zonal flows (super rotation) and eddies embedded in them, is forced by the differential heating between poles and equator and affected by external factors such as the rotation rate of the earth and boundary conditions including the orography. To understand how the main features of the general circulation come about, the responses of the general circulation to changes in the main forcing factors have been studied by varying the strength of the forcing factors. For instance, the effect of the earth's rotation rate on the general circulation is studied by varying the earth's rotation rate in a numerical model (HIDE, 1977; HUNT, 1979a, 1979b; DEL GENIO and SuOzZO, 1987; NAVARRA and BocCALETTI, 2002). The effect of the differential heating was first studied using the laboratory experiments by FULTZ et al.

\footnotetext{
*Corresponding author: Jin-Song von Storch, Max-Planck-Institut für Meteorologie, Bundesstrasse 53, 20146 Hamburg, Germany, e-mail: jin-song.storch@dkrz.de
}

(1959) and has been reexamined using an atmospheric GCM by STENZEL and VON STORCH (2004). Different circulation regimes, characterized by different values of the axial component of the global relative angular momentum, are found when varying the strength of the differential forcing. These regimes resemble those obtained by varying the earth's rotation rate, although the reasons for the circulation changes are different.

One question, which belongs to the class of problems considered by the previous studies, is what is the effect of the orography on the general circulation. Different to the differential heating and the earth's rotation rate, whose influence is expected to be global, orography is often considered as a local forcing factor. For instance, a recent study (SCHNEIDER, 2000) using the same atmospheric model considered here deals with the effect of the orography on the interactions between two idealized stormtracks, whereby leaving the impact of the orography on the general circulation characterized by global indices, such as the global atmospheric angular 
momentum (AAM), unconsidered. The most prominent study concerning the effect of topography may be the work by CHARNEY and DEVORE (1979) who considered the non-linear interactions with topography as a cause for the emergence of multiple flow equilibria associated with blocking. Despite of conceptual value of their work, the model they have used is too simple to deal with the effect of orography on the global features of the general circulation.

This paper aims to understand the impact of a meridionally oriented barrier on the global circulation characterized by the axial component of the relative AAM, $M_{r}$. $M_{r}$ is defined by

$$
M_{r}=\int_{V} \rho u a \cos (\varphi) d V
$$

where $\int_{V} d V$ indicates the global volume integral, $u$ is the zonal velocity, $a$ is the earth's radius and $\rho$ is the density of air. In the absence of extraterrestrial influence, the balance equation of the vertically integrated and zonally and temporally averaged absolute angular momentum reads, in the sigma coordinates,

$\frac{1}{g} \int_{0}^{1} \frac{\partial}{\partial t}\left[\overline{p_{s} m_{a}}\right] d \sigma=0$

$$
\begin{array}{r}
=\underbrace{-\frac{1}{g a \cos \varphi} \int_{0}^{1} \frac{\partial}{\partial \varphi} \mathscr{F}(\varphi) \cos \varphi d \sigma}_{A} \\
\underbrace{-\frac{a}{g} \cos \varphi\left[\overline{p_{s} F_{\lambda}}\right]}_{B}-\underbrace{\left[\overline{\left.p_{s} \frac{\partial h}{\partial \lambda}\right]}\right.}_{C},
\end{array}
$$

where $m_{a}=(\Omega a \cos \varphi+u) a \cos \varphi$ is the absolute angular momentum per unit mass, and

$$
\mathscr{F}(\varphi)=a^{2} \cos ^{2}(\varphi) \Omega\left[\overline{p_{s} v}\right]+a \cos \varphi\left(\left[\bar{u} \overline{p_{s} v}\right]+\left[\overline{u^{\prime}\left(p_{s} v\right)^{\prime}}\right]\right)
$$

is the meridional flux of absolute angular momentum. $F_{\lambda}$ is the zonal component of the divergence of the stress tensor, $h$ the elevation of the earth's surface, $p_{s}$ surface pressure and $v$ the meridional velocity. Following the notation of PEIXOTO and OORT (1992), the zonal average of a variable $x$ is denoted by $[x]$, the time mean by $\bar{x}$, the deviations from $\bar{x}$ by $x^{\prime}$. Eq. (1.1) states that under the stationary condition, the vertically integrated divergence of the angular momentum flux $\mathscr{F}(\varphi)($ term $A$ ) must balance the zonally averaged friction (term $B$ ) and mountain torques (term $C$ ). Eq. (1.1) will be used to analyze the changes in the angular momentum budget induced by orographic features.

It will be shown that an orographic barrier not only produces large disturbances in the immediate neighborhood of the barrier, it also changes the global relative

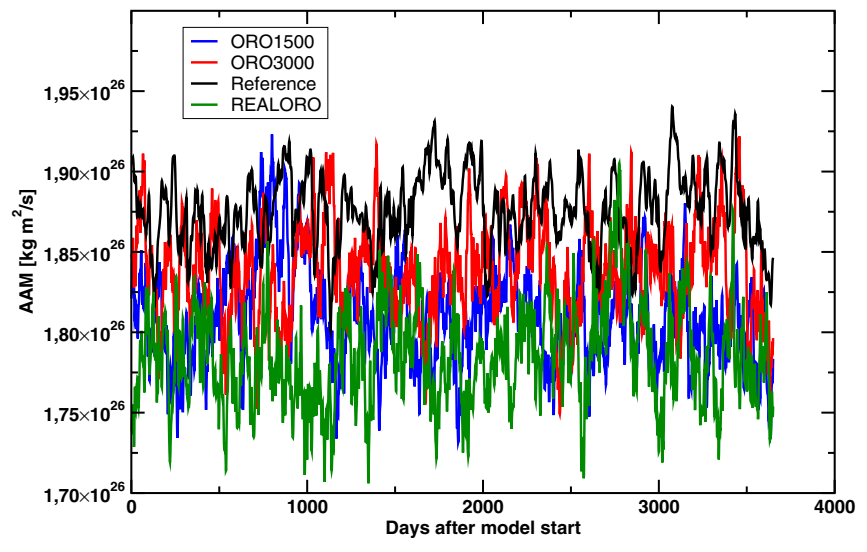

Figure 1: The global relative angular momentum in the three experiments with orography, ORO1500 (blue), ORO3000 (red), REALORO (green), and the reference-run without orography (black).

AAM in a non-linear manner. Section 2 describes the experiments performed to isolate these effects. The results of the experiments are analyzed in the light of angular momentum budget in section 3. A summary is given in the final section.

Table 1: Means and standard deviations of the global relative atmospheric angular momentum, $M_{r}$, in the reference run and the three experiments with different orographic features.

\begin{tabular}{|l|c|c|}
\hline experiment & $\begin{array}{c}\text { mean } \\
\left(10^{26} \mathrm{~kg} \mathrm{~m}^{2} / \mathrm{s}\right)\end{array}$ & $\begin{array}{c}\text { standard deviation } \\
\left(10^{24} \mathrm{~kg} \mathrm{~m}^{2} / \mathrm{s}\right)\end{array}$ \\
\hline reference & 1.88 & 2.3 \\
\hline ORO1500 & 1.81 & 3.02 \\
ORO3000 & 1.84 & 3.00 \\
REALORO & 1.78 & 3.01 \\
\hline
\end{tabular}

\section{PUMA experiments}

The effect of a meridionally oriented orographic barrier is studied using the Portable University Model of the Atmosphere (PUMA) developed at the university of Hamburg (FRAEDRICH et al., 1998). The hydrodynamics of the model are essentially identical to the ECHAM model (ROECKNER et al., 1996), but the diabatic and dissipation processes are described by much simpler parametrisations than those in the ECHAM model. The processes in the boundary layer are parametrized by Rayleigh friction in the equations of vorticity and divergence in the lowest model layer. The diabatic processes are parametrized by a relaxation toward a prescribed radiative equilibrium temperature field. A standard version of PUMA uses a radiative equilibrium temperature field which corresponds to the equinox condition and has an equator-pole difference of $60 \mathrm{~K}$. Such a version, implemented at the T21 resolution using 5 vertical layers without orography, will be used to obtain a 


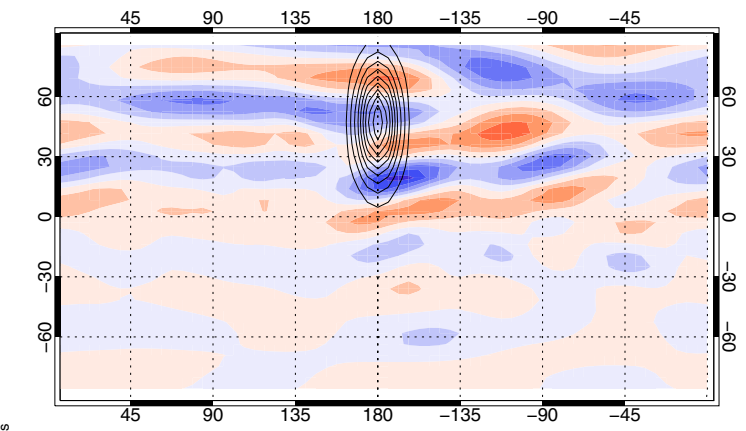

a)

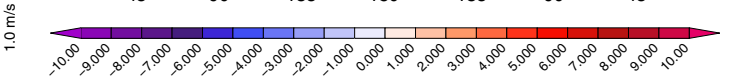

(a)

b)

Figure 2: Differences in the time-mean $300 \mathrm{hPa}$ zonal velocity (experiment minus reference) in $\mathrm{ms}^{-1}$ between a) experiment ORO1500 and the reference run, and b) experiment ORO3000 and the reference run. The black contours mark the lines equal surface geopotential for a) experiment ORO1500 and b) experiment ORO3000. The intervals between two isolines is $1338 \mathrm{~m}^{2} \mathrm{~s}^{-1}$ for a) and $2675 \mathrm{~m}^{2} \mathrm{~s}^{-1}$ for b).

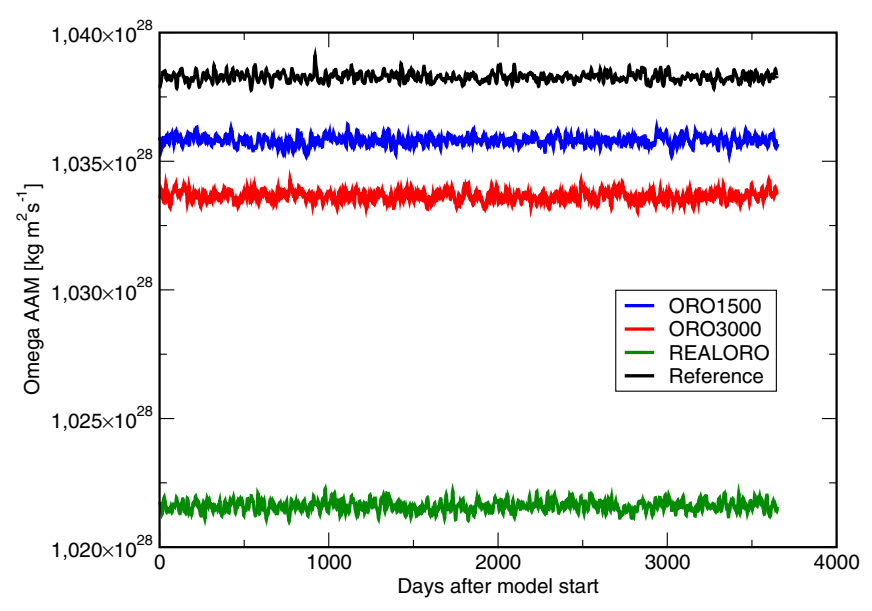

Figure 3: The global omega angular momentum in the three experiments, ORO1500 (blue), ORO3000 (red), REALORO (green), and the reference-run (black).

reference run. As shown by FRAEDRICH et al. (1998), the atmosphere simulated by this version of PUMA captures realistic features of the synoptic eddies.

To study the effect of orography on the global circulation, a meridionally oriented orographic barrier is built into the model. The structure of the barrier is given by

$$
z(\varphi, \lambda)=Z \exp \left(-\left[\frac{\left(\varphi-\varphi_{0}\right)^{2}}{b^{2}}+\frac{\left(\lambda-\lambda_{0}\right)^{2}}{c^{2}}\right]\right)
$$

which was used by SCHNEIDER (2000). $\varphi$ and $\lambda$ denote the latitude and longitude. $z(\varphi, \lambda)$ has a twodimensional Gaussian distribution with the maximum being located at $\left(\varphi_{0}, \lambda_{0}\right)=\left(45^{\circ} N, 180^{\circ}\right)$ and the exponential decay rate being $(b, c)=\left(11.5^{\circ}, 28.125^{\circ}\right)$. With these constants one obtains an orographic barrier whose latitudinal extent is larger than the longitudinal extent (see contour lines in Fig. 2). The height of the barrier, $Z$, is set to 1500 meter in the first and 3000 meter in the second experiment. The two experiments are hereafter referred to ORO1500 and ORO3000, respectively.
For the comparison, a third experiment (experiment REALORO) using realistic topography is carried out. The topography is obtained by interpolating the Terrain Base $^{1}$ data onto the model grid with the T21 resolution and five vertical layers. As a result of the interpolation, the topography used is flatter than that in the real world.

The standard version of PUMA without orography and the PUMA with orography given in Eq. (2.1) with two different heights and with the realistic orography are integrated each for 10 years. These four runs will be analyzed in the next section.

\section{The effect of orography on the global relative AAM}

\section{a) The primary effect}

When introducing orography into the system, two changes in the global relative AAM are found. The first one concerns the mean value of $M_{r}$. Fig. 1 shows the daily time series of $M_{r}$, calculated according to Eq. (1.1) using data from the reference run (black), and the experiments ORO1500, ORO3000 and REALORO (blue, red and green), respectively. $M_{r}$ obtained from the reference run tends to be larger than those obtained from ORO3000, ORO1500 and REALORO. This tendency is further confirmed by the mean values of $M_{r}$ given in Tab. 1. A reduction in the mean value of $M_{r}$ is found in all three orography experiments, even though the magnitudes differ from experiment to experiment. Also shown in Tab. 1 are the standard deviations of $M_{r}$. The differences between any two means are larger or comparable to the typical values of one standard deviation, indicating the statistical significance of the differences. Physically, a difference of about $10^{25} \mathrm{~kg} \mathrm{~m}^{2} / \mathrm{s}$, as found between experiment REALORO and the reference run, corresponds to a change in the length of the day of about

\footnotetext{
${ }^{1}$ NOAA, National Geophysical Data Center
} 


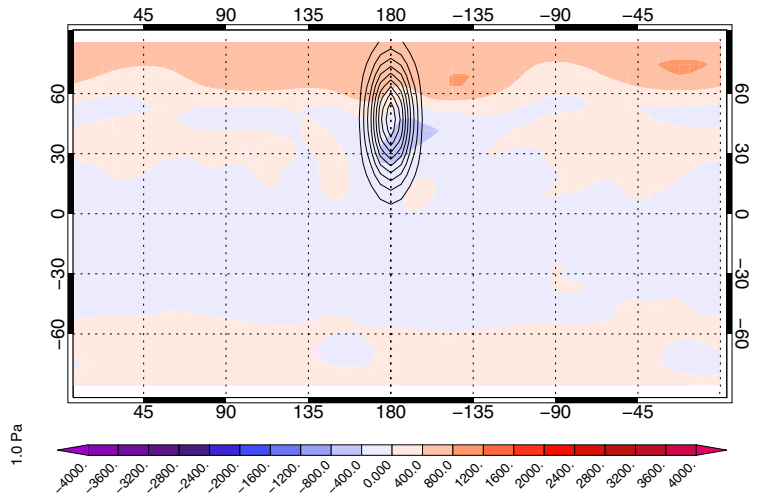

a)

Figure 4: Differences in the time-mean sea level pressure (experiment minus reference) in Pa between a) experiment ORO1500 and the reference run, and b) experiment ORO3000 and the reference run. The black contours mark the lines equal surface geopotential for a) experiment ORO1500 and b) experiment ORO3000. The intervals between two isolines is $1338 \mathrm{~m}^{2} \mathrm{~s}^{-1}$ for a) and $2675 \mathrm{~m}^{2} \mathrm{~s}^{-1}$ for b).

$0.17 \mathrm{~ms}$, which is about $20 \%$ of the observed change induced by the annual cycle. The atmosphere rotates slower with orography than without orography.

A consideration of the time-averaged zonal wind at $300 \mathrm{hPa}$ shows that the smaller values of $M_{r}$ results from the breaking of zonal symmetry, rather than an overall decrease in zonal winds. When differences in the zonal velocity between experiments and the reference run are calculated (Fig. 2), one finds that the largest wind maxima are located downstream of the orographic features. The strict zonal symmetry of the zonal wind in the reference run (not shown) disappears in the experiments.

The second change concerns the variability. Relative to the reference run, $M_{r}$ in the experiments with orography has a much larger variance (Tab. 1). The variance of $M_{r}$ is proportional to the mean kinetic energy of the time-varying part of the zonal flow. Thus, an increase in variance suggests an enhancement of the kinetic energy in the time-varying part of the zonal flow. In the presence of orography, the energy inputed into the PUMAatmosphere is not only stored in the time-mean zonal flow, but also in the time-varying zonal flow.

Although the present paper concentrates on the relative angular momentum, the omega angular momentum (the part of the absolute angular momentum that is related to the atmospheric mass) is also considered for the sake of completeness. The omega angular momentum is strongly reduced in the presence of orographic features (Fig. 3). The reduction in experiment ORO3000 is stronger than that in experiment ORO1500. The largest reduction is found when the realistic topography is implemented. Fig. 4 shows the difference in the mean sea level pressure between experiment ORO1500 and the reference run (top) and between ORO3000 and the reference run (bottom). The most striking feature is the increase of pressure at the northern high latitudes and the decrease of pressure in the tropics and the subtropics, indicating a polward shift of mass. It is this shift of mass that leads to the decrease of the omega angular momentum. Despite of its large impact on the absolute value of the angular momentum, the polward mass shift is not directly responsible for the changes in the relative angular momentum shown in Fig. 1 and will therefore not be considered further. As will be discussed in section $3 b$, only the stripes of positive and negative pressure anomalies at midlatitudes between $30-60^{\circ} \mathrm{N}$ are dynamically relevant for the change in the relative angular momentum.

To conclude, the primary effect of orography on the relative angular momentum is the symmetry breaking. Associated with this symmetry breaking is a decrease in the mean of $M_{r}$ accompanied by an increase in the variance of $M_{r}$.

\section{b) The effect involving the eddy-mean-flow interaction}

If the symmetry breaking is the only effect induced by an orographic barrier, one would expect that the higher the orographic barrier, the larger is the reduction in the mean of $M_{r}$. This is however not the case. According to Tab. 1 the mean value of $M_{r}$ in ORO3000 is larger, rather than smaller than that in ORO1500.

To understand why a higher orographic barrier leads to an increase rather than a decrease of $M_{r}$, the balance of the vertically integrated and zonally averaged angular momentum in the experiments and the reference run is analyzed. The contribution of the meridional mass transport $\left[\overline{p_{s} v}\right]$ to the angular momentum flux $\mathscr{F}(\varphi)$ in Eq. (1.3) vanishes if there is no accumulation of mass at latitude $\varphi$ over time. This condition is hard to meet by a GCM. It is not exactly fulfilled in the PUMA model (STENZEL, 2004) nor in many other atmospheric GCMs (KEITH, 1995). Nonetheless, there is, at least in the PUMA model, no accumulation of the error in the mass imbalance over time (STENZEL, 2004). This suggests that one may leave out the contribution of mass 


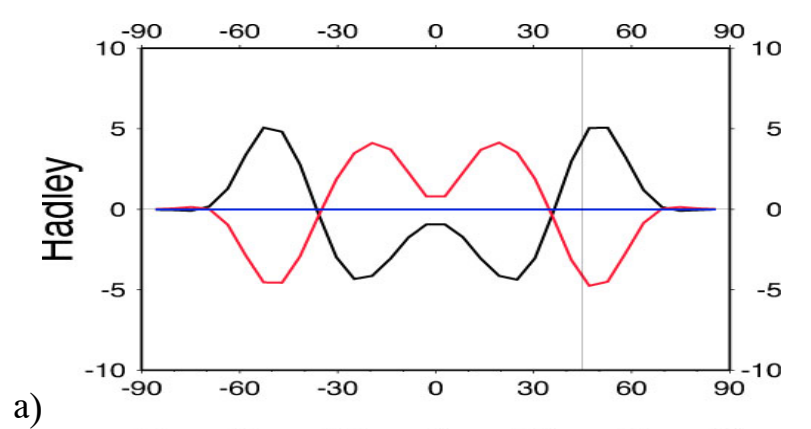

a)

b)
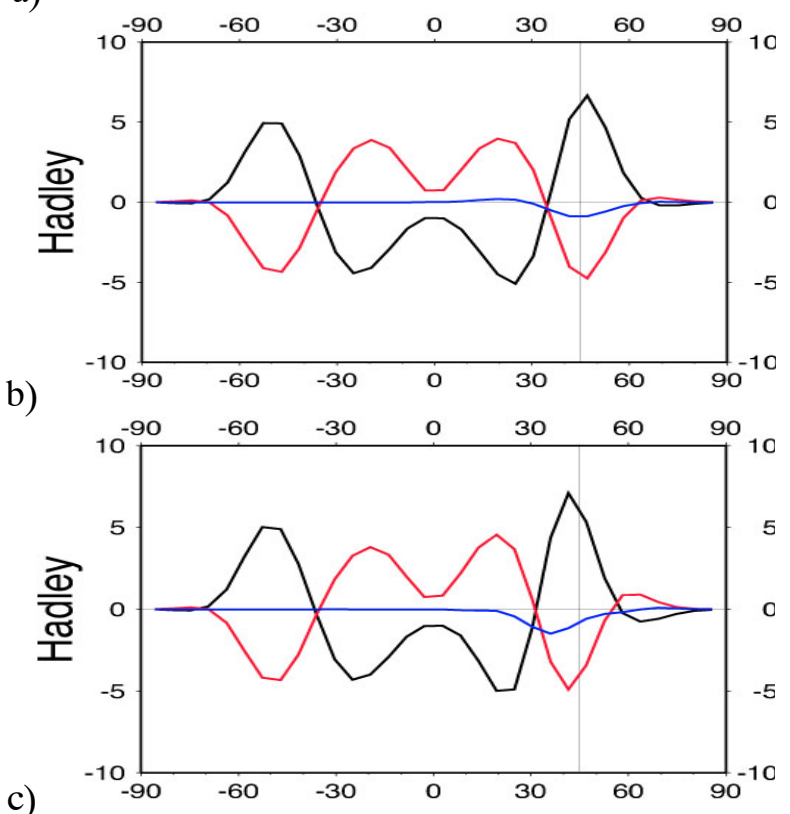

c)

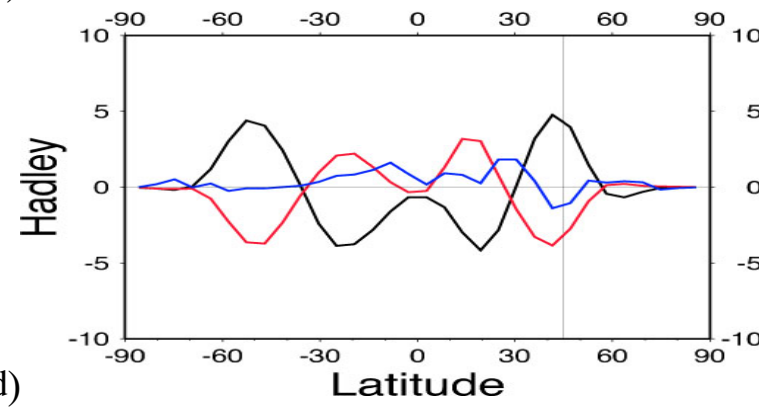

Figure 5: Angular momentum balance, given in Hadleys (1 Hadley $=10^{18} \mathrm{~kg} \mathrm{~m}^{2} \mathrm{~s}^{-2}$ ) for a) the reference run, b) experiment ORO1500, c) ORO3000 and d) REALORO. The latitudinal profiles of the three terms in Eq. (1.1), the divergence of angular momentum flux (term $A$ ), the friction torque (term $B$ ) and the mountain torque (term $C$ ), are plotted in black, red and blue respectively.

to $\mathscr{F}$ when considering the time-mean balance, even when this contribution does not vanish. Fig. 5 shows the meridional profiles of the three terms on the right hand side of Eq. (1.1). Term $A$ does not include the contribution from $\left[\overline{p_{s} v}\right]$. The vertical line marks $45^{\circ} \mathrm{N}$ where the center of the orographic barrier in ORO1500 and ORO3000 is located. Overall, the three terms $A, B$ and $C$ in Eq. (1.1) balance each other at each latitude.
Consider first the change in the angular momentum balance induced by a low barrier by comparing the angular momentum balances obtained from the reference run and experiment ORO1500. In the reference run with no orography, the term $C$ in Eq. (1.1) is zero. The vertically integrated divergence of $\mathscr{F}$ (term $A$ ) balances the friction torque (term $B$ ). The maximum of the vertical integral of the divergence of $\mathscr{F}$ is located at about $50^{\circ}$. In experiment ORO1500, the orographic barrier centered at $45^{\circ}$ induces a negative mountain torque with the maximum at essentially the same latitude as the barrier. This torque is balanced by a change in the meridional gradient of vertically integrated and zonally averaged angular momentum flux $\mathscr{F}$. The result is an equatorward shift of the center of the maximum divergence of $\mathscr{F}$ from $50^{\circ} \mathrm{N}$ in the reference run (black curve in Fig. 5a) to about $46^{\circ} \mathrm{N}$ in experiment ORO1500 (black curve in Fig. 5b).

According to Eq. (1.3), $\mathscr{F}$ contains contributions from the time-mean flow, $\bar{u} \overline{p_{s} v}$, and the transients, $\overline{u^{\prime}\left(p_{s} v\right)^{\prime}}$. A further examination of eddy fields in the reference run and experiment ORO1500 suggests that the contribution from transients dominates the change in the divergence of $\mathscr{F}$. Fig. 6a shows the standard deviation of the $500-\mathrm{hPa}$ geopotential height in experiment ORO1500 relative to that in the reference run. The dipole with large negative values north of the large positive values at about $45^{\circ} \mathrm{N}$ suggests that the synoptic variability is shifted southward and enhanced at about $45^{\circ} \mathrm{N}$ in experiment ORO1500 relative to that in the reference run. It is this change in eddy activities which leads to a change in the divergence of eddy momentum flux and from that to the change in the divergence of vertical integrated and zonally averaged angular momentum flux shown in Fig. 5 b.

The divergence of the eddy momentum flux in turn acts as a forcing for the zonal-mean flow (see e.g. KAROLY, 1990; HARTMANN and LO, 1998). This forcing changes the zonal-mean zonal flow in two ways. First, the zonal-mean zonal winds are strengthened at the center of the divergence of $\mathscr{F}$. These winds are geostrophically balanced by the pressure anomalies at midlatitudes shown in Fig. 4a. The positive anomalies near $60^{\circ} \mathrm{N}$ and the negative ones at about $40^{\circ} \mathrm{N}$ produces the required pressure gradient. Secondly the location of the zonal-mean zonal wind is shifted equatorward, relative to its position in the reference run, implying an increase in the distance of the zonal flow to the rotation axis. Both changes lead to an increase in $M_{r}$. In other words, the change in the transients induced by the orographic barrier acts to increase the global relative AAM.

The above described changes in the global relative AAM in experiment ORO1500 is further enhanced in experiment ORO3000 with a higher orographic barrier. Compared with experiment ORO1500, the mountain torque is enhanced and has its maximum further 

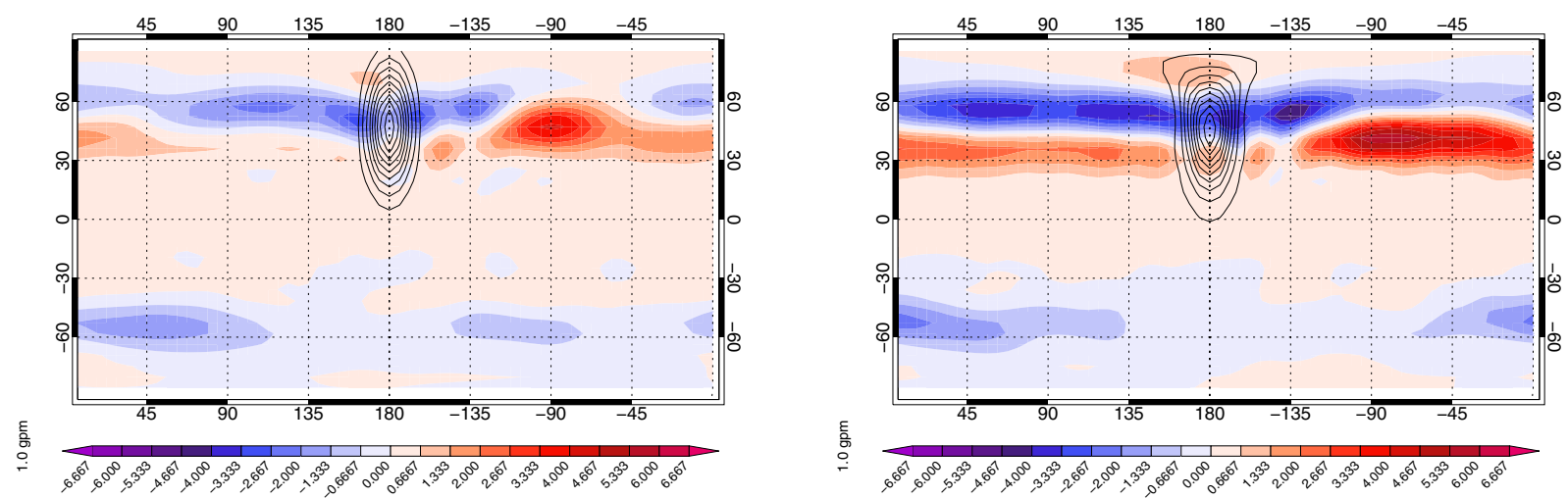

a)

Figure 6: Differences of the standard deviation of 2-to-6 day bandpass-filtered geopotential height at $500 \mathrm{hPa}$ (experiment minus reference) in gpm between a) experiment ORO1500 and the reference run, and b) experiment ORO3000 and the reference run. The black contours mark the lines equal surface geopotential for a) experiment ORO1500 and b) experiment ORO3000. The intervals between two isolines is $1338 \mathrm{~m}^{2} \mathrm{~s}^{-1}$ for a) and $2675 \mathrm{~m}^{2} \mathrm{~s}^{-1}$ for b).

equatorward (blue line in Fig. 5c). This change in mountain torque is related to a trough downstream of the orographic barrier (Fig. 4) which is not only much more pronounced but also extends further equatorward relative to that in experiment ORO1500. The resulting mountain torque is balanced by a divergence of $\mathscr{F}$ (black curve in Fig. 5c) whose maximum is located further equatorward relative to that in experiment ORO1500. The divergence of $\mathscr{F}$ induces an eddy forcing that enhances zonal-mean zonal wind and shifts the zonal wind further equatorward and hence further away from the rotation axis relative to that in experiment ORO1500. The result is an even stronger increase in $M_{r}$ in ORO3000 than in ORO1500.

The angular momentum balance in experiment REALORO is also shown to illustrate that the PUMA implemented with a realistic orography is able to produce a mountain torque resemble that derived from the observation (PEIXOTO and OORT 1992). Consistent with the torque shown by PEIXOTO and OORT, the mountain torque in the bottom panel in Fig. 5 shows positive values in the tropics and subtropics and negative ones at mid-latitudes, in particular in the Northern Hemisphere.

\section{Conclusions}

The effect of an orographic barrier on the global circulation is quantified by the axial component of the global relative angular momentum, $M_{r}$. The primary effect is a breaking of zonal symmetry by the orographic barrier. Such a breaking allows more energy to be stored in the time-varying part of the zonal flow and leads therefore to a circulation which is characterized by a smaller mean value of $M_{r}$ relative to the case without orographic features.

In addition to the breaking of zonal symmetry, there is a second effect which leads to an increase in the mean value of $M_{r}$. An orographic barrier induces generally a non-zero mountain torque. This torque is essentially balanced by a change in the divergence of the angular momentum flux. Associated with such a change is an eddy forcing that enhances the zonal-mean zonal winds and shifts the zonal-mean zonal winds equatorward and hence further far away from the rotation axis. As a result, one finds an increase in the mean value of $M_{r}$.

The effect associated with the eddy-mean-flow interaction is further strengthened when the height of the orographic barrier is increased. Thus, when the zonal symmetric flow is perturbed by an orographic barrier of different height in experiments ORO1500 and ORO3000, one finds generally a decrease in the mean value of $M_{r}$ due the symmetry breaking effect. However, as the effect associated with the eddy-mean-flow interaction is stronger in the presence of a higher orographic barrier, the decrease in the mean of $M_{r}$ is smaller in experiment ORO3000 than in experiment ORO1500.

Apart from the effects on the relative AAM, orographic barriers produces also a polward shift of mass, leading a striking decrease of omega angular momentum. This feature is not directly responsible for the changes in the relative angular momentum. Further investigations are required to clarify whether or not the mass shift is a specific feature of the PUMA model, and if not, what is the physical mechanism responsible for the shift.

\section{Acknowledgements}

This work is supported by the project "Die Atmosphäre und ihr Drehimpuls" funded by Deutsche Forschungsgemeinschaft (STO 377/2).

\section{References}

Charney J.G., J. G. Devore, 1979: Multiple flow equilibria in the atmosphere and blocking. - J. Atmos. Sci. 36, 1205-1216. 
Del Genio, A. D., R. J. Suozzo, 1987: A comparative study of rapidly and slowly rotating dynamical regimes in a terrestrial general circulation model. - J. Atmos. Sci. 44, 973-986.

FRAEDRICH, K., E. KIRK, F. LUNKEIT, 1998: Portable university model of the atmosphere. - Technical report $\mathbf{1 6}$. DKRZ, Hamburg, $37 \mathrm{pp}$.

Fultz, D., R. R. Long, G. V. Owens, W. Bohan, R. KAYLOR, J. WeIL, 1959: Studies of thermal convection in a rotating cylinder with some implications for large-scale atmospheric motions. - Meteor. Monographs 4,1-104.

HARTMANN, D.L., F. Lo, 1998: Wave-driven zonal flow vacillation in the Southern Hemisphere. - J. Atmos. Sci. 55, 1303-1315.

HIDE, R., 1977: Experiments with rotating fluids. - Quart. J. Roy. Met. Soc. 103 (435), 1-28.

HUNT, B. G., 1979 a: The effects of past variations of the Earth's rotation rate on climate. - Nature 281, 188-191.

—, 1979 b: The influence of the Earth's Rotation Rate on the General Circulation. - J. Atmos. Sci. 36, 1392-1408.

KAROLY, D.J., 1990: The role of transient eddies in lowfrequency zonal variations of the Southern Hemisphere circulation. - Tellus, 42A, 41-50.

KeITH, D.W., 1995: Meridional Energy-Transport - Uncertainty in Zonal Means. - Tellus 47, 30-44.
Navarra, A., G. BocCAletti, 2002: Numerical general circulation experiments of sensitivity to Earth rotation rate. - Climate Dyn. 19, 467-483.

PeiXOTTO, J.P., A. H. OORT, 1992: Physics of climate. American Institute of Physics, 520 pp.

Roeckner, E., L. Arpe, L. Bengtsson, M. Christoph, M. Claussen, L. DÜMenil, M. Esch, M. Giorgetta, U. SCHLESE, U. SCHUlzWEIDA, 1996: The atmospheric general circulation model ECHAM-4: Model description and simulation of present-day climate. - MPI-Report 218, $90 \mathrm{pp}$.

SCHNEIDER, H., 2000: Einfluß der Orographie auf die Wechselwirkung zweier Stormtracks in einem vereinfachten globalen Zirkulationsmodell. - Diplomarbeit, Universität Hamburg, Meteorologisches Institut, 105 pp.

STENZEL, O., 2004: Dynamische und thermodynamische Einflüsse auf den Drehimpuls der Atmosphäre und dessen Auswirkung auf die Allgemeine Zirkulation. - Doktorarbeit, Universität Hamburg, Meteorologisches Institut, 102 pp.

STEnzel, O,. J.-S. VON STORCH, 2004: On the effect of thermal forcing on the global AAM and the general circulation. - Climate Dyn. 22, 415-427. 